\title{
SMOOTH RETRACTS OF EUCLIDEAN SPACE
}

\author{
GOO ISHIKAWA AND TAKASHI NISHIMURA
}

\begin{abstract}
A characterization of a $C^{s}$ submanifold of Euclidean space which can become a $C^{r}$ retract of some $C^{r}$ retraction, $(0 \leqq r \leqq s \leqq \infty)$, is given.
\end{abstract}

\section{Introduction}

A $C^{r}$ endomorphism $f: \boldsymbol{R}^{n} \rightarrow \boldsymbol{R}^{n}$ is called a $C^{r}$ retraction if it satisfies the property $f=f \circ f$. For a $C^{r}$ retraction $f$, its image $f\left(\boldsymbol{R}^{n}\right)$ is called a $C^{r}$ retract. It is well-known that any $C^{r}$ retract is a $C^{r}$ submanifold of $\boldsymbol{R}^{n}$ without boundary if $r \geqq 1$ (for instance, see [B, H]).

The purpose of this paper is to characterize when a connected $C^{s}$ submanifold of $\boldsymbol{R}^{n}$ can be realized as a $C^{r}$ retract $(0 \leqq r \leqq s \leqq \infty)$ as follows.

In this paper, submanifolds are always regular submanifolds.

Definition. Let $M$ be a connected $C^{s}$ submanifold of $\boldsymbol{R}^{n}(0 \leqq s \leqq \infty)$. We say $M$ is $C^{r}$ contractible in itself $(0 \leqq r \leqq s)$, if there exists a $C^{r}$ mapping

$$
\phi: M \times[0,1] \longrightarrow M
$$

such that $\phi(, 0)$ is the identity mapping and $\phi(, 1)$ is a constant mapping.

THEOREM. Let $s$ be a fixed integer $\geqq 0$ or $\infty$. For a connected $C^{s}$ submanifold $M$ of $\boldsymbol{R}^{n}$ without boundary $(0 \leqq s \leqq \infty)$, the following $(2 s+2)$ conditions are equivalent.

$\left(a_{r}\right) \quad M$ is a $C^{r}$ retract of some $C^{r}$ retraction $(0 \leqq r \leqq s)$,

$\left(b_{r}\right) \quad M$ is closed in $\boldsymbol{R}^{n}$ and $C^{r}$ contractible in $M(0 \leqq r \leqq s)$.

By this theorem, every image of $\boldsymbol{R}^{1}$ closedly $C^{\infty}$ embedded in $\boldsymbol{R}^{3}$ (i. e. open $C^{\infty}$ knot) can be realized as a $C^{\infty}$ retract of some $C^{\infty}$ retraction. Furthermore, there is the socalled Newman-Whitehead 3-dimensional manifold, which is an open manifold hence a $C^{\infty}$ manifold and $C^{0}$ contractible in itself but not homeomorphic to $\boldsymbol{R}^{3}[\mathrm{~N}-\mathrm{Wd}]$. If we embed this 3 -dimensional manifold closedly in $55 \mathrm{M} 99$.

1980 Mathematics Subject Classification (1985 revision). Primary 58C25; Secondary

Received November 29, 1993. 
$\boldsymbol{R}^{6}$ by using of Whitney's embedding theorem [Wy], then this theorem asserts that even this embedded $C^{\infty}$ manifold can be realized as a $C^{\infty}$ retract of some $C^{\infty}$ retraction.

The authors do not know whether or not the similar result holds for a connected $C^{\omega}$ submanifold. Furthermore, there are many $C^{\omega}$ submanifolds of $\boldsymbol{R}^{n}$ which are $C^{0}$ contractible in themselves but can not be realized as algebraic retracts (remark that the intersection of an algebraic retract and an affine subspace is a semi-algebraic subset of $\boldsymbol{R}^{n}$ by Tarski-Seidenberg theorem, so, the number of its connected components is finite). Hence, it seems interesting to investigate how we can characterize $C^{\omega}$ submanifolds of $\boldsymbol{R}^{n}$ which can be realized as algebraic retracts.

In the complex case, holomorphic retracts were already being studied (for instance, see $[R]$ ). However, it seems that they were being investigated only in the local case to characterize singularities of analytic spaces. So, it seems to be significant to propose the similar question in the complex case.

\section{$\S 1$. An interpretation of smooth retracts}

Consider the Euclidean space $\boldsymbol{R}^{n}$ as the space of the state of an animal brain. A smooth endomorphism $f: \boldsymbol{R}^{n} \rightarrow \boldsymbol{R}^{n}$ can be considered as the smooth operation of learning an object. Then, we can consider the property $f=f \circ f$ of a smooth retraction $f$ means the equivalence between learning an object once and doing it twice successively. That is to say, smooth retractions represent the most primitive objects which an animal never forget if once he learns them; and smooth retracts are, therefore, the sets of states after learning the most primitive objects.

Alternatively, if we regard a smooth endomorphism $f$ as a model of "stimulation", then smooth retractions represent the most primitive stimulations which an animal become experienced if once he receives them.

The authors consider that also from the viewpoint of this interpretation smooth retracts are interesting.

For any integers $k, r, s$ with $0 \leqq r \leqq s \leqq \infty$ and $1 \leqq k \leqq \infty$, let $\mathcal{S}_{r}^{s}(k)$ be the set of all $C^{s}$ submanifolds of $\boldsymbol{R}^{n}$ which can be realized as images $f\left(\boldsymbol{R}^{n}\right)$ of some $C^{r}$ endomorphisms $f: \boldsymbol{R}^{n} \rightarrow \boldsymbol{R}^{n}$ with the property $f^{k}=f^{k+1}$, where $f^{l}$ means the $l$ times composition of $f, f^{l}=\underbrace{f \circ f \circ \ldots \circ f}_{l \text { times }}$; and we regard any $C^{r}$ endomorphism $f: \boldsymbol{R}^{n} \rightarrow \boldsymbol{R}^{n}$ has the trivial property $f^{\infty}=f^{\infty}$.

Trivially, we have

$$
\begin{gathered}
\mathcal{S}_{0}^{s}(k) \supset \mathcal{S}_{1}^{s}(k) \supset \cdots \supset \mathcal{S}_{s}^{s}(k), \\
\mathcal{S}_{r}^{s}(1) \subset \mathcal{S}_{r}^{s}(2) \subset \mathcal{S}_{r}^{s}(3) \subset \cdots \subset \mathcal{S}_{r}^{s}(\infty) \quad \text { and } \\
\mathcal{S}_{r}^{r}(k) \supset \mathcal{S}_{r}^{r+1}(k) \supset \cdots \supset \mathcal{S}_{r}^{s}(k) ;
\end{gathered}
$$


for any $k, r, s$ with $0 \leqq r \leqq s \leqq \infty$ and $1 \leqq k \leqq \infty$. We can easily see that for any $s$ with $0 \leqq s \leqq \infty$ and any connected $C^{s}$ submanifold $M$ of $\boldsymbol{R}^{n}, M$ is included in the set $\mathcal{S}_{s}^{s}(\infty)$. Our theorem asserts that

$$
\mathcal{S}_{0}^{s}(1)=\mathcal{S}_{1}^{s}(1)=\cdots=\mathcal{S}_{s}^{s}(1),
$$

for any $s$ with $0 \leqq s \leqq \infty$.

From the above viewpoint, the second primitive objects can be considered those which are realized as images of some smooth endomorphisms $f: \boldsymbol{R}^{n} \rightarrow \boldsymbol{R}^{n}$ with the property $f^{2}=f^{3}$. The following simple example shows that

$$
\mathcal{S}_{r}^{s}(1) \neq \mathcal{S}_{r}^{s}(2)
$$

for any $r, s$ with $0 \leqq r \leqq s \leqq \infty$. Hence, even for the set $\mathcal{S}_{r}^{s}(2)$ with $0 \leqq r \leqq s \leqq \infty$, characterizing also it seems to be interesting.

Example. Let $\gamma: \boldsymbol{R} \rightarrow[0,1] C^{\infty}$ be a non-decreasing function such that

$$
\gamma(t)=0 \quad \text { if } t \leqq 4 .
$$

Then set the $C^{\infty}$ mapping $h: \boldsymbol{R}^{2} \rightarrow \boldsymbol{R}^{2}$ as follows :

$$
h(x, y)=\gamma\left(x^{2}+y^{2}\right)(x, y) .
$$

Let $k: \boldsymbol{R}^{2} \rightarrow \boldsymbol{R}^{2}$ be the projection to the second component $k(x, y)=(0, y)$. Identify $\boldsymbol{R}^{2}$ with $\boldsymbol{C}$ and let $l: \boldsymbol{C}=\boldsymbol{R}^{2} \rightarrow \boldsymbol{R}^{2}=\boldsymbol{C}$ be the $C^{\omega}$ mapping with the form $l(z)=$ $\exp (z)-1$. Then put $f=l \circ k \circ h: \boldsymbol{R}^{2} \rightarrow \boldsymbol{R}^{2}$.

Since $h \circ l \circ k(x, y)=0$ for any $(x, y) \in \boldsymbol{R}^{2}$ and $l \circ k(0)=0$, we have $f^{2}\left(\boldsymbol{R}^{2}\right)=\{0\}$. Therefore $f^{2}=f^{3}$.

However from the construction above, we see $f\left(\boldsymbol{R}^{2}\right)=S^{1}$. Hence, $S^{1}$ is included in the set $S_{\infty}^{\infty}(2)$. From (1.1) and (1.3), $S^{1}$ is included in the set $S_{r}^{s}(2)$ for any $r, s$ with $0 \leqq r \leqq s \leqq \infty$.

On the other hand, from our theorem, $S^{1}$ is not included in the set $S_{r}^{s}(1)$ for any $r, s$ with $0 \leqq r \leqq s \leqq \infty$.

\section{§2. Proof of theorem}

Trivially, we have

and

$$
\left(a_{s}\right) \Rightarrow\left(a_{s-1}\right) \Rightarrow \cdots \Rightarrow\left(a_{1}\right) \Rightarrow\left(a_{0}\right)
$$

$$
\left(b_{s}\right) \Rightarrow\left(b_{s-1}\right) \Rightarrow \cdots \Rightarrow\left(b_{1}\right) \Rightarrow\left(b_{0}\right) .
$$

We divide the proof of theorem into the following three steps.

(Step 1) $\left(b_{r}\right)$ implies $\left(a_{r}\right) \quad(0 \leqq r \leqq s)$.

(Step 2) $\left(a_{r}\right)$ implies $\left(b_{r}\right) \quad(0 \leqq r \leqq s)$. 
(Step 3) (bo) implies $\left(b_{s}\right)$.

Proof of step 1. Let $\phi: M \times[0,1] \rightarrow M$ be a $C^{r}$ contraction mapping such that $\phi(, 0)$ is the identity mapping and $\phi(x, 1)=x_{0}$ far all $x \in M$. Since $M$ is a $C^{s}$ submanifold, it is a $C^{s}$ neighborhood retract; i.e., there are a neighborhood $U_{M}$ of $M$ in $\boldsymbol{R}^{n}$ and a $C^{s}$ retraction $\rho: U_{M} \rightarrow M$. Take a $C^{s}$ function $\varepsilon: M \rightarrow(0,1]$ so that for each $x \in M$ the ball of radius $\varepsilon(x)$ around $x$ is contained in $U_{M}$. Take also a $C^{\infty}$ bump function $\lambda:[0,1] \rightarrow[0,1]$ such that

$$
\lambda(t)= \begin{cases}1 & \text { if } 0 \neq t<\frac{1}{3} \\ 0 & \text { if } \frac{2}{3}<t \neq 1 .\end{cases}
$$

We set the $C^{r}$ mapping $\Phi: U_{M} \rightarrow M$ as follows :

$$
\Phi(x)=\phi(x, \lambda(\|x-\rho(x)\| / \varepsilon(\rho(x))))
$$

for any $x \in U_{M}$. Then the restriction of $\Phi$ to the subset

$$
\tilde{U}_{M}=\left\{x \in U_{M} \mid \frac{2}{3} \varepsilon(\rho(x))<\|x-\rho(x)\| \leqq \varepsilon(\rho(x))\right\}
$$

of $U_{M}$ is the constant mapping $\tilde{U}_{M} \rightarrow\left\{x_{0}\right\}$.

Since the submanifold $M$ is closed in $\boldsymbol{R}^{n}$, we have

$$
\operatorname{cl}\left(\boldsymbol{R}^{n}-U_{M}\right) \cap \operatorname{cl}\left(U_{M}\right) \subset \operatorname{cl}\left(\tilde{U}_{M}\right),
$$

where $\operatorname{cl}(X)$ means the topological closure of $X$ in $\boldsymbol{R}^{n}$.

Hence we can extend the mapping $\Phi$ to a $C^{s}$ mapping

$$
\widetilde{\Phi}: \boldsymbol{R}^{n} \longrightarrow M
$$

as $\tilde{\Phi}\left(\boldsymbol{R}^{n}-U_{M}\right)=\left\{x_{0}\right\}$.

Since the restriction $\tilde{\Phi}$ to $M$ is the identity mapping, the mapping $\tilde{\Phi}: \boldsymbol{R}^{n} \rightarrow M$ must be a $C^{r}$ retraction.

Proof of step 2. This step is easy. In fact, we can see step 2 applies in analytic, algebraic situation also.

Let $f: \boldsymbol{R}^{n} \rightarrow M$ be a $C^{r}$ retraction. Since the restriction $\left.f\right|_{M}$ is the identity mapping, $M$ must be closed in $\boldsymbol{R}^{n}$. We set $C^{s}$ mapping $\phi: \boldsymbol{R}^{n} \times[0,1] \rightarrow M$ as $\phi(x, t)=f((1-t) x)$. Then $\phi(, 0)$ is $f$ and $\phi(, 1)$ is the constant mapping. Thus, $M$ is a $C^{r}$ contractible in itself.

Proof of step 3. Let $\phi: M \times[0,1] \rightarrow M$ be a continuous contraction mapping such that $\phi(, 0)$ is the identity mapping and $\phi(x, 1)=x_{0}$ for all $x \in M$. Take a chart $(\phi, U)$ such that $x_{0} \in U$ and $\phi\left(x_{0}\right)=0 \in \boldsymbol{R}^{k}$. Since $M$ is a $C^{s}$ manifold, $\phi$ is a $C^{s}$ mapping. Let $\mu:[0,1] \rightarrow[0,1]$ be the function as follows : 


$$
\mu(t)= \begin{cases}2 t & \text { if } 0 \leqq t \leqq \frac{1}{2} \\ 1 & \text { if } \frac{1}{2} \leqq t \leqq 1\end{cases}
$$

By using of the approximation theorem $[\mathrm{H}]$, we can approximate the mapping $\phi(x, \mu(t))$ by a $C^{s}$ mapping

$$
\tilde{\phi}: M \times[0,1] \longrightarrow M
$$

such that $\tilde{\phi}(M \times[1 / 2,1]) \subset U$.

Since we can take an approximation mapping $\tilde{\phi}$ so that $\tilde{\phi}(, 0)$ is a diffeomorphism of $M$, there exists an inverse mapping $\tilde{\phi}^{-1}(, 0)$ which is also $C^{s}$ mapping. Using the same bump function $\lambda: R \rightarrow[0,1]$ of step 1 , set the $C^{s}$ mapping $\Phi: M \times[0,1] \rightarrow M$ as follows :

$$
\Phi(x, t)= \begin{cases}\tilde{\phi}\left(\tilde{\phi}^{-1}(x, 0), t\right) & \text { if } 0 \leqq t \leqq \frac{1}{2} \\ \phi^{-1}\left(\lambda(2 t-1)\left(\psi\left(\tilde{\phi}\left(\tilde{\phi}^{-1}(x, 0), t\right)\right)\right)\right) & \text { if } \frac{1}{2} \leqq t \leqq 1\end{cases}
$$

Then, we can see that $\Phi(, 0)$ is the identity mapping and $\Phi(x, 1)=x_{0}$ for all $x \in M$.

Therefore, $M$ is $C^{s}$ contractible.

Acknowledgements. The authors wish to thank S. Izumi and E. Yoshinaga for their useful suggestion.

\section{REFERENCES}

[B] TH. BRöcker, Differentiable Germs and Catastrophes, London Math. Soc. Lecture Note Ser., 17, Cambridge University Press, Cambridge, 1975.

[H] M.W. HiRsch, Differential Topology, Graduate Texts in Math., 33, SpringerVerlag, New York-Heidelberg-Berlin, 1976.

[N-Wd] M.H. A. Newman and J.H.C. Whitehead, On the group of a certain linkage, Quart. J. Math., 8 (1937), 14-21.

[R] H. Rossi, Vector fields on analytic spaces, Ann. of Math., 78 (1963), 455-467.

[Wy] H. Whitney, The selfintersection of a smooth $n$-manifold in $2 n$-space, Ann. of Math., 45 (1944), 220-246.

Department of Mathematics

Faculty of Science

HOKKAIDO UNIVERSITY

SAPPORO 060

JAPAN 
Department of Mathematics

Faculty of Education

Yokohama National University

Hodogaya-KU, YoKohama 240

JAPAN 